\title{
Sealing Ability of Two Different Furcal Perforation Repair Materials with and without Internal Matrix: An In Vitro Study
}

\author{
Pooja Keshrani ${ }^{1}$, Nimisha Shah ${ }^{2}$, Nishtha Patel $^{3}$, Anjali Oak ${ }^{4}$, Kamal Bagda ${ }^{5}$, Payal Patel ${ }^{6}$
}

\begin{abstract}
Aim: To evaluate the sealing ability of mineral trioxide aggregate (MTA) and resin-modified glass ionomer cement (RMGIC) to seal the furcal perforation with and without internal matrix.

Materials and methods: Sixty freshly extracted intact human permanent mandibular molars were selected. After creating furcal perforation, the teeth were randomly divided into four experimental groups containing 15 teeth each according to the furcal perforation repair materials used. Group I-RMGIC without internal matrix. Group II—RMGIC with internal matrix. Group III—MTA without internal matrix. Group IV—MTA with internal matrix. To evaluate the sealing ability of furcal perforation, dye extraction method was performed using $2 \%$ methylene blue dye and $65 \%$ concentrated nitric acid. Spectrophotometer was used for measuring dye absorbance at $550 \mathrm{~nm}$.

Results: Group I (RMGIC without internal matrix) showed highest microleakage followed by group II (RMGIC with internal matrix), group III (MTA without internal matrix), and group IV (MTA with internal matrix). There was a significant difference found between group I and group II, but there was no significant difference seen between group III and group IV.

Conclusion: Mineral trioxide aggregate has excellent sealing ability and can be used with and without internal matrix in repair of furcation perforation. Resin-modified glass ionomer cement should be used with internal matrix to repair furcation perforations.

Clinical significance: Mineral trioxide aggregate with and without internal matrix whereas RMGIC with internal matrix have been successfully used in repair of furcation perforation.
\end{abstract}

Keywords: Furcation perforation, ProGide, ProRoot MTA.

World Journal of Dentistry (2019): 10.5005/jp-journals-10015-1657

\section{INTRODUCTION}

In today's era, the world has undergone a complete revolution under endodontic treatment due to the public's demand of RCT over tooth extraction. Endodontics plays a significant role in raising the standard of oral health. Endodontic mishaps occur due to inattention to detail or totally unpredictable. ${ }^{1}$

Right from the diagnosis to the termination of the treatment, at each and every step of endodontic procedure there are the chances of the occurrence of endodontic mishaps. Every endodontist has experienced variety of emotions ranging from the thrill-of-the fill to various procedural accidents. ${ }^{2,3}$

Among the all, perforation is the second most common cause of root canal failure. Perforation can be defined as direct communication between the tooth and the periodontium. There are two types of perforations: pathological and iatrogenic. ${ }^{4-8}$

A perforation results due to various reasons such as malposition teeth, variant internal tooth anatomy, operator's inexperience. ${ }^{9}$ Perforation may occur at any level of the tooth. It was the worst possible outcome in root canal treatment. ${ }^{1}$ latrogenic furcal perforation is due to misdirection of bur during endodontic treatment. ${ }^{9,10}$

Prognosis for a tooth with a perforation depends on the site and size of the perforation, periodontal condition, contamination time, sealing ability of the perforation, sterility of the canal, biocompatibility of material, accessibility to the main canal, etc. Generally, it can be said that the sooner the repair is undertaken, the better the chance of success. ${ }^{11,12}$ Different types of materials have been used for perforation repair. ${ }^{4}$

The most commonly used repair materials are amalgam, glass ionomer cement, IRM super-EBA, resin-glass ionomer hybrids, composite resins, MTA, etc. ${ }^{13}$
1,3,4,6 Department of Conservative Dentistry and Endodontics, College of Dental Sciences and Research Centre, Manipur, Ahmedabad, Gujarat, India

${ }^{2}$ Department of Conservative Dentistry and Endodontics, KM Shah Dental College and Hospital, Vadodara, Gujarat, India

${ }^{5}$ Department of Conservative Dentistry and Endodontics, Narsinhbhai Patel Dental College and Hospital, Visnagar, Gujarat, India

Corresponding Author: Nishtha Patel, Department of Conservative Dentistry and Endodontics, College of Dental Sciences and Research Center, Manipur, Ahmedabad, Gujarat, India, Phone: +91 9712994610, e-mail: dr.nkp.2557@gmail.com

How to cite this article: Keshrani P, Shah N, Patel N, et al. Sealing Ability of Two Different Furcal Perforation Repair Materials with and without Internal Matrix: An In Vitro Study. World J Dent 2019;10(5):359-363.

Source of support: Nil

Conflict of interest: None

Resin-modified glass ionomer cement has been promoted as a material with pronounced antibacterial activity, low cytotoxicity, and favorable tissue response and good chemo-mechanical bonding. It has been used for sealing of the furcal perforation over the conventional GIC as they have faster setting time. It sets immediately after light curing. It has less microleakage because of limited moisture sensitivity, minimal curing shrinkage, and greater depth of penetration of polymer into the tooth surface, etc. ${ }^{14}$ The data concerning the efficacy of this material are limited. 
Recently, a new perforation repair and root end filling material, MTA, has been introduced at the Loma Linda University by Torabinejad. $^{15}$

The main constituents of MTA are tricalcium aluminate, tricalcium silicate, tricalcium oxide, silicate oxide, mineral oxide, and bismuth oxide. It is non-resorbable, nontoxic, radiopaque, and very biocompatible and sets in the presence of moisture with a $\mathrm{pH}$ of $12.5 .{ }^{15}$ The sealing ability of MTA has been shown to be superior than amalgam, zinc-oxide eugenol, resin-modified GIC, and resin materials. Also, cytotoxicity of MTA has been found to be less than IRM or Super EBA. ${ }^{16}$ The excellent and unique property of MTA is that it promotes cementum regeneration, thus facilitating the PDL regeneration. ${ }^{13}$

Major problem arises during the sealing of perforation with any material is that there are chances of extrusion of sealing material into the periodontal apparatus and bone that can cause inflammatory reactions and delayed healing. To prevent this, Lemon in 1992 internal matrix concept was developed by for perforation repair. The internal matrix is made up of either collagen, hydroxyapatite, or calcium sulfate. All these materials are biocompatible and resorbable within few days. The internal matrix is cut into small pieces and condensed at the perforation site after which the sealing material is layered against the internal matrix until the perforation is sealed. This internal matrix reconstructs the outer shape of the root, facilitates the adaptation of the repair material, and prevents the in-growth of the epithelial tissue which allows the formation of normal periodontal ligament. It prevents the extrusion of the sealing material, reconstructs the outer shape of the root, and helps in better healing. ${ }^{17}$

Thus, the study was planned to evaluate the sealing ability of MTA and RMGICs in furcal perforation repair, with and without the use of an internal matrix by dye-extraction method.

\section{Materials and Methods}

Sixty freshly extracted human intact permanent first and second mandibular molars extracted purely for periodontal reasons free of caries, cracks, fractures, restorations with non-fused and nonhypoplastic teeth were selected.

First and second mandibular molars were mounted in polyvinyl siloxane impression material (Affinious Precious, Whaldent, California). Endodontic access cavity was prepared by high-speed long shank round bur no. 4 (Mani Inc., Japan) for initial entry followed by long-tapered non-end cutting bur (TR-13; Mani Inc., Japan) for lateral wall extension with the use of the high-speed hand piece (NSK Inc., Japan). Orifices of the canals were negotiated and sealed with temporary filling material (Cavit-MD Temp; Meta Biomed Co Ltd, Korea).

Perforation was created in the furcal area between mesial and distal orifices using a high-speed long shank round bur no. 4 (Mani Inc., Japan) (Fig. 1). Double coat of clear nail varnish (Sunshine Cosmetics, Metoda, India) was applied over the cavity walls and pulpal floor apart from $1 \mathrm{~mm}$ around the margin of the perforation in each specimen (Fig. 2).

All specimens were randomly divided into four experimental groups to compare the sealing ability of different materials (15 samples in each group).

Group I-resin-modified GIC without internal matrix

Group II-resin-modified GIC with internal matrix

Group III-MTA without internal matrix

Group IV-MTA with internal matrix
In groups II and IV, internal matrix (ProGide-B. V. Medical Technologies, Holland) was cut $2 \mathrm{~mm}$ more than perforation size and placed to the furcal area by Buchanan Hand Pluggers (Sybron endo) (Fig. 3). Resin-modified GIC (GC Corporation Tokyo, Japan) and ProRoot MTA were manipulated according to the manufacturer's recommendation. They were kept on the perforation site in increments with the microapical placement system (Products Dentaries SA, Vevey, Switzerland) and condensed with Buchanan Hand Pluggers (Sybron endo). Moist cotton was kept over MTA to allow setting of MTA (Fig. 4).

Specimens were placed in larger size $(2 \mathrm{~mL})$ Eppendorf tubes (Eppendorf, North America) with moist cotton to simulate the clinical condition. Molars were fixed with the tubes by cyanoacrylate adhesive (Fevikwik; Pidilite Industries Ltd, India).

After sealing of perforation with MTA, resin-modified GIC specimens were placed for 24 hours in 100\% humidity. $2 \%$ of methylene blue dye (Merck Specialities Private Limited, Mumbai, India) was applied inside the access cavity of all samples for 24 hours for checking the microleakage by dye extraction method. Once the dye immersion was completed, the specimens were thoroughly washed under tap water for half an hour to remove all residues of methylene blue. After molars were transferred in sterile



Fig. 1: Access cavity preparation

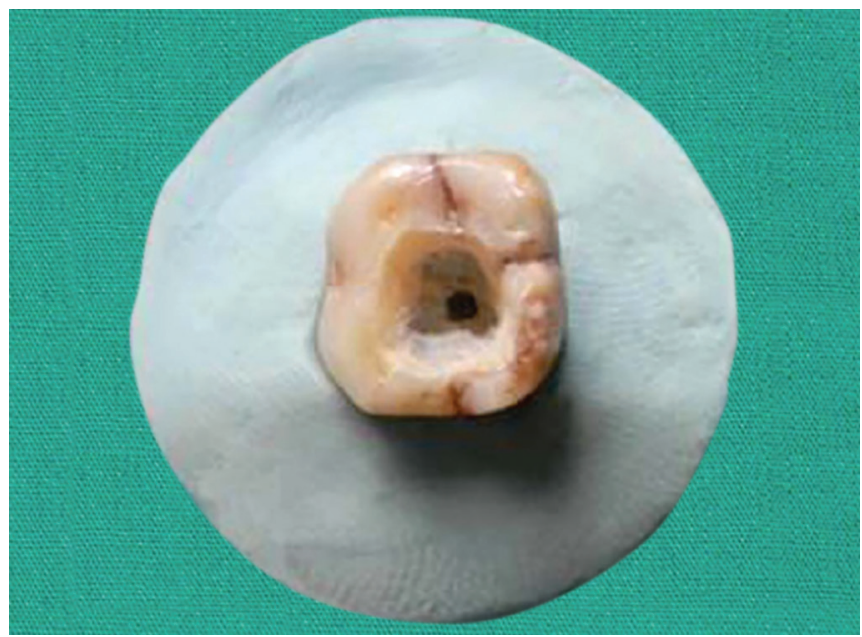

Fig. 2: Furcation perforation, sealing with cavity 




Fig. 3: Schematic representation of groups I and III specimens

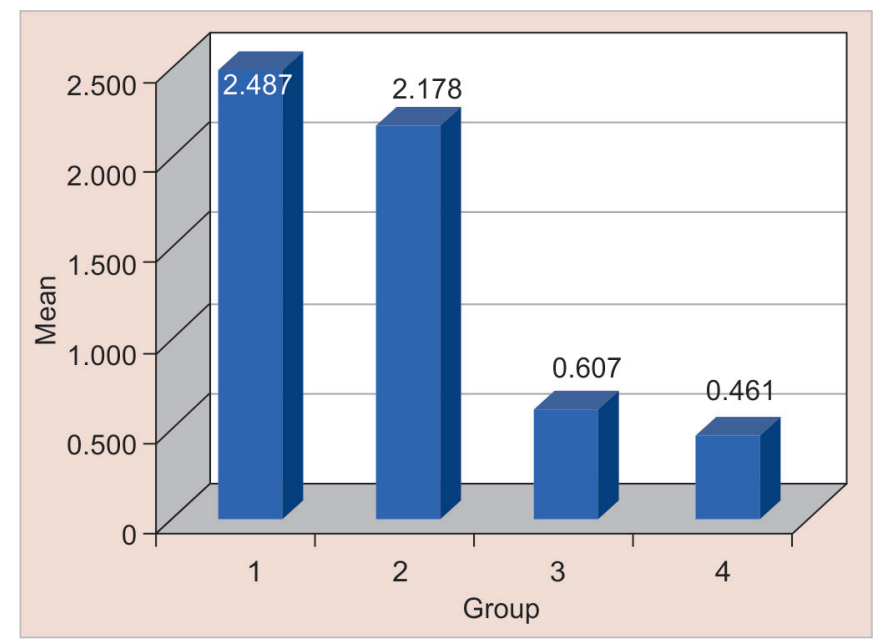

Fig. 5: Comparison of mean values between all groups

container containing $65 \%$ nitric acid (SDFI Industrial Estate, Mumbai) for 3 days. Centrifugation was done at 14,000 rpm for 5 minutes to separate debris from the extracted dye. $200 \mu \mathrm{L}$ of supernatant was transferred in Eppendorf tubes (Eppendorf, North America). Then, spectrophotometer was used for measuring dye absorbance at $550 \mathrm{~nm}$ wavelength.

Statistical analysis was carried out with SPSS 14.0 (trial version) software. The descriptive statistical analysis was done by ANOVA test. When ANOVA test was significant, a post hoc test was performed for pair-wise comparison ( $p \leq 0.05)$.

\section{Results}

On comparing mean values, it was seen that group I (2.487) showed highest microleakage followed by group II (2.178), group III (0.606), and group IV (0.433). Groups I and II were shown a significant difference, but there was no significant difference between groups III and IV (Fig. 5).

\section{Discussion}

According to Ingle, perforation is the second most common reason for endodontic failure and this accounts for $9.6 \%$ of all endodontic failures. ${ }^{1}$ During the non-surgical re-treatment procedures,

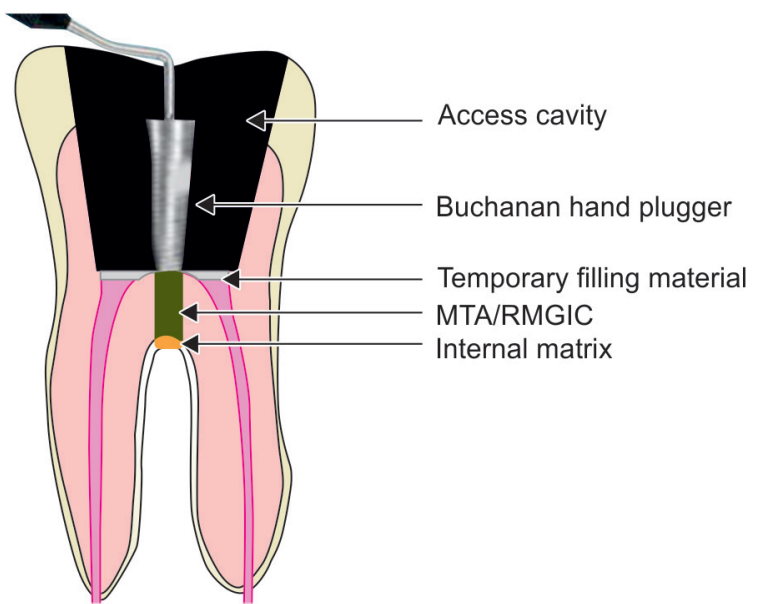

Fig. 4: Schematic representation of groups II and IV specimens

perforations were found to be present in 7-12 percent of the previous endodontic treatments. ${ }^{13}$

Kvinnsland et al. found that post-insertion iatrogenic perforations occur in $57 \%$ of cases; mostly, high percentage of perforations occur in furcation areas of multirooted teeth during the removal of dentin from the pulp chamber floor while searching of root canals were $47 \%$. Other causes of furcal perforation are anatomic variations of tooth, inaccessibility of the tooth, and inexperience of the operator. ${ }^{18}$

Periodontium inflammation occurs due to furcal perforations of the in-multirooted teeth, which can lead to an irreversible loss of periodontal attachment. ${ }^{4}$ The first indication of an iatrogenic perforation is the evidence of persistent bleeding into the pulp space.$^{14}$ Immediate treatment of the perforation should be carried out for a favorable prognosis. ${ }^{4,8}$

Sealing ability of perforation materials and to re-establish a healthy periodontal ligament are two important factors for better prognosis of endodontic treatment. ${ }^{18}$ The properties of an ideal furcal perforation repair material should provide good hermetic seal, it should be noncontaminated by hemorrhage, it should be biocompatible, nonresorbable, insoluble, should prevent its extrusion into PDL apparatus, should promote osteogenesis and cementogenesis, should be non-toxic, non-carcinogenic, easily available, convenient to the patient or dentist, and non-expensive. ${ }^{17}$

In this study, MTA and resin-modified GIC were selected to seal the furcal perforation due to its better biocompatibility, sealing ability, and proper adaptability to the dentinal walls as compared to other materials.

MTA was first available in grey color. The use of this material was limited to the confines of the canal and/or the pulp chamber area below the crestal bone level as the material led to discoloration. Recently, a new type of MTA is available, which is off-white in color. White MTA is used as a substitute for grey MTA in anterior teeth, as it has a hue that matches more closely to that of natural tooth color. The difference in color between the two types of MTA is due to the difference in their compositions. The grey-colored MTA contains tetra calcium aluminoferrite in its composition. The lack of this iron containing compound accounts for the off-white appearance of white MTA. The better handling properties of white MTA are due to the absence of larger and elongated particles. ${ }^{19}$ 
Initially, MTA was used in surgical endodontic treatment for root end filling, but now, its clinical uses have expanded, which comprises pulp capping, apexification, pulpotomy, perforation repair, etc. ${ }^{20}$

Ford et al. were the first investigators who used MTA for repair of furcal perforations. They showed that regeneration of cementum was done in furcation perforation cases, which were repaired with MTA than amalgam. ${ }^{4}$

Mineral trioxide aggregate consists of very small hydrophilic particles capable of setting in the moisture environment. Its composition includes tricalcium silicate, tricalcium aluminate, tricalcium oxide, silicate oxide, and tetra calcium alumina ferrite with small additions of other metal oxides such as bismuth oxide. Electron probe microanalysis of MTA powder showed calcium and phosphorus ions, which are the main constituents of hard tissues. ${ }^{19}$ Camilleri et al. proved that both forms of MTA has excellent biocompatibility while in contact with cells and tissues..$^{21}$ The quality of aggregate is based on the size of particles, water-powder ratio, temperature, presence of moisture. ${ }^{15,22}$

Immediately after mixing, $\mathrm{pH}$ of MTA is 10.2, and after 3 hours, it increases up to 12.5; then it remains constant..$^{15}$ The high alkalinity of MTA enables it to induce hard tissue formation when used for perforation repair. Lee et al. evaluated the effect of physiological environment on the hydration behavior and physical properties of MTA. An acidic environment as would be present with infection or suppuration must be cleared up before application of MTA because acidic environment such as $\mathrm{pH} 5$ is detrimental compared to $\mathrm{pH} 7 .^{20}$

Torabinejad et al. suggested that a wet cotton pellet placed over MTA for 24 hours. A study showed the effects of setting condition on flexural strength of white MTA. Two-sided moisture is always more effective than one-sided moisture, which shows more flexural strength after 24 hours. $^{15}$

Another important property of perforation repair material is push out strength because function of tooth might displace the material after perforation repair. Moisture is important factor for the push out strength of MTA. So, MTA receives optimal physical properties such as compressive, flexural, and push-out strength when it gets enough moisture after the placement of material in a perforation site. ${ }^{15}$

When MTA was mixed with distilled water in the 3:1 ratio, it results in the formation of a colloidal gel, which solidifies and creates hard structure in less than 3 hours. MTA consists of two specific phases: crystalline calcium oxide and amorphous calcium phosphate. When MTA comes in contact with the tissue fluids, calcium oxide reacts with water to form calcium hydroxide. ${ }^{21}$

$$
\mathrm{CaO}+\mathrm{H}_{2} \mathrm{O} \rightarrow \mathrm{Ca}(\mathrm{OH})_{2}
$$

The formation of a hard structure when MTA is used in perforation repair is due to the reaction of calcium hydroxide with carbon dioxide resulting in the production of calcite crystals.

$$
\mathrm{Ca}(\mathrm{OH})_{2}+\mathrm{CO}_{2} \rightarrow \mathrm{CaCO}_{3} \text { (calcite crystal) }
$$

The reaction explaining the adaptability of MTA to dentine is as follows: In contact with the tissue fluids, MTA dissolves releasing all of its major cationic elements $\left(\mathrm{Ca}^{2+}, \mathrm{Mg}^{2+}\right)$, which triggers the precipitation of hydroxyapatite on its surface. ${ }^{21}$

$10 \mathrm{Ca}^{2+}+6\left(\mathrm{PO}_{4}\right)^{3-}+2(\mathrm{OH})^{1-} \rightarrow \mathrm{Ca}_{10}\left(\mathrm{PO}_{4}\right)^{6}(\mathrm{OH})^{2}$ (hydroxyapatite)
These hydroxyapatite crystals grow filling the microscopic spaces between the MTA and the dentinal wall. As time progresses, a diffusion-controlled mechanism between the apatite layer and dentine leads to chemical bonding. ${ }^{21}$

Dual-cured resin-modified GIC are those glass ionomer materials that are modified by the inclusion of resin, generally to make them partly photo-curable. Setting of RMGIC is a combination of two reactions: it sets through resin polymerization from exposure to visible light, then additional hardening by an acid base reaction. ${ }^{14}$

Advantages of RMGIC's over conventional GIC includes sufficient long working time controlled in command to a snap set by photo curing, improved setting characteristics, protection of the acid base reaction from problem of water balance, rapid development of early strength, early finishing and polishing after immediate set, improved chemical and mechanical bonding with tooth structure, fluoride release is comparable to conventional glass ionomer cement and greater than compomers, high diametral strength (20 MPa), high fracture toughness, higher moisture resistance, and higher sealing ability. Because of these advantages, RMGIC is considered as one of the furcal perforation repair material. ${ }^{14}$

Always use freshly mixed sealing material with soft consistency and placed without pressure. Then, internal matrix is used as a barrier material to push the granulation tissue out of the perforation site and keep it at that site only.

ProGide has two distinctive surface textures: The outer surface is dense, and inner surface is porous. The dense outer surface in contact with the soft tissue is cell occlusive, and it prevents epithelial down growth. So, it minimizes the attachment of gingival connective tissues with the healing bone surface. Simultaneously, macromolecular pore size allows for nutrient transfer. The porous and roughened surface of bone allows a growth at a cellular level. When this surface is comes in contact with the bone, it stimulates cellular osteoblastic integration surface and stabilizing the blood clot. It comes in $20 \mathrm{~mm} \times 25 \mathrm{~mm}$ or $25 \mathrm{~mm} \times 30 \mathrm{~mm}$ sizes. ${ }^{23}$

Hashem and Hassanien conducted a study to evaluate the sealing ability of two types of MTA (ProRoot MTA and MTAAngelus) and intermediate restorative material (IRM) with and without internal matrix to seal large furcal perforations using a dye-extraction method. Intermediate restorative material without internal matrix showed the highest dye absorbance when ProRoot MTA with and without internal matrix and MTA Angelus with internal matrix showed the least dye absorbance. ${ }^{22}$

Various methods of detecting microleakage are dye penetration method, dye extraction method, fluid filtration technique, use of color-producing microorganisms, radioactive isotopes such as $45 \mathrm{Ca}$, 1311, 35S, 22Na, air pressure method, electrochemical methods, scanning electron microscopy, thermal and mechanical cycling, and neutron activation analysis. Dye penetration is the oldest method among all these. But there are some drawbacks of dye penetration including size of molecule that is smaller than bacteria, which cannot measure the actual absorbed value but it only measures the deepest part reached by the dye. To overcome this disadvantage, nowadays dye extraction method and fluid filtration techniques are used, which are based on quantitative measurements of the passage of liquid within the interfaces. Although the fluid filtration technique is considered as a gold standard method for checking microleakage, dye extraction technique presents a great advantage over the fluid-filtration technique. The fluid-filtration technique is very time-consuming, costly, and technique-sensitive, and required 
specialized equipment to perform the same, whereas dye extraction method is simple, very less time-consuming, less-expensive, as effective as fluid-filtration technique and required equipments are available in most of the universities. So, in this study we evaluated the sealing ability of various perforation repair materials by dye extraction method. ${ }^{23}$

Absorbance was checked by spectrophotometer which is based on the principal of wavelength. If the material has less sealing ability, dye leakage is more and absorbance is more and vice versa. ${ }^{24}$

Krupalini et al. compared the sealing ability of medicated calcium sulfate, hydroxyapatite, and MTA as a furcal perforation repair material. The results showed that MTA can be used with and without internal matrix due to least microleakage and best sealing ability where RMGIC should be used with internal matrix to repair the furcation perforation. ${ }^{25}$

In this study, result showed that group I (RMGIC without internal matrix) showed highest microleakage followed by group II (RMGIC with internal matrix), group III (MTA without internal matrix), and group IV (MTA with internal matrix). There was a significant difference between groups I and II, but there was no significant difference between groups III and IV.

Within the limitations of the study, the evaluation of the clinical performance of the materials in vivo is needed to give reliable recommendations for dentists.

\section{Conclusion}

On the basis of the results of this study, MTA has excellent sealing ability, which can be used with or without internal matrix, whereas RMGIC should be used with internal matrix to repair furcal perforations.

\section{References}

1. Ingle Jl, Bakland KI. Endodontics, 5th ed, London: BC Decker Inc.; 2002.

2. Saini D, Saini R. Accidents in endodontics: a case report. J Nep Dent Assoc 2009;10(1):61-64.

3. Lsmann MH. Mechanical preparation of root canals: shaping goals, techniques and means. Endod Topics 2005;12(1):52-70. DOI: 10.1111/j.1601-1546.2005.00194.x.

4. Ford TR, Torabinejad M, McKendry DJ, et al. Use of mineral trioxide aggregate for repair of furcal perforations. Oral Surg Oral Med Oral Pathol Oral Radiol Endod 1995;79(6):756-762. DOI: 10.1016/S10792104(05)80313-0.

5. Snyder WR, Hoover J, Khoury J, et al. Effect of agents used in perforation repair on osteoblastic cells. J Endod 1997;23(3):158-161. DOI: 10.1016/S0099-2399(97)80266-X.

6. Fuss Z, Trope M. Root perforations: classification and treatment choices based on prognostic factors. Endod Dent Traumatol 1996;12(6):255-264. DOI: 10.1111/j.1600-9657.1996.tb00524.x.
7. Louise $\mathrm{H}$, Scott L, et al. American association of endodontists, glossary of endodontic terms, 7th ed., Chicago, IL; 2003.

8. Cauder T, Shin S. Repair of perforations with MTA: clinical applications and mechanisms of action. Endod Topics 2009;15(1):32-55. DOI: 10.1111/j.1601-1546.2009.00242.x.

9. Grossman LI, Oliet S, Del CE. Endodontic practice, 11th ed., 1988.

10. Sinai IH. Endodontic perforations: their prognosis and treatment. J Am Dent Assoc 1977;95(1):90-95. DOI: 10.14219/jada.archive. 1977.0531.

11. Eldeeb ME, Eldeeb M, Tabibi $A$, et al. An evaluation of the use of amalgam, cavit and calcium hydroxide in the repair of furcation perforations. J Endod 1982;8(10):459-466. DOI: 10.1016/S00992399(82)80151-9.

12. Aguirre R, Eldeeb ME. Evaluation of the repair of mechanical furcation perforations using amalgam, gutta-percha or indium foil. J Endod 1986;12(6):249-256. DOI: 10.1016/S0099-2399(86)80256-4.

13. Ruddle JC. Nonsurical endodontic treatment. In Cohen S, Burns RC, ed. Pathways of the pulp, 8th ed. St Louis: Mosby Inc.; 2002. p. 919.

14. Alhadainy HA, Abdalla Al. Artificial floor technique used for the repair of furcation perforations: a microleakage study. J Endod 1998;24(1):33-35. DOI: 10.1016/S0099-2399(98)80209-4.

15. Torabinejad M, Hong CU, Mcdonald F, et al. Physical and chemical properties of a new root end fillin material. J Endod 1995;21(7): 349-353. DOI: 10.1016/S0099-2399(06)80967-2.

16. Daoudi MF, Saunders WP. In vitro evaluation of furcal perforation repair using MTA or RMIC with and without the use of operating microscope. J Endod 2002;28(7):512-515. DOI: 10.1097/00004770200207000-00006.

17. Lemon RR. Nonsurgical repair of furcation defects (internal matrix concept). Dent Clin North Am 1992;36(2):439-457.

18. Kvinnsland I, Oswald RJ, Hasle A, et al. A clinical roentgenological study of 55 cases of root perforation. Int Endod J 1989;22(2):75-84. DOI: 10.1111/j.1365-2591.1989.tb00509.x.

19. Lee $Y L$, Lee $B S$, Lin FH, et al. Effects of physiological environments on the hydration behavior of mineral trioxide aggregate. Biomaterials 2004;25(5):787-793. DOI: 10.1016/S0142-9612(03)00591-X.

20. Nishtha $P$, Kiran $P$, Suheel MB, et al. Comparing gray and white mineral trioxide aggregate as a repair material for furcation perforation: an in vitro dye extraction study. J Clin Diagn Res 2014;8(10):ZC70-ZC73. DOI: $10.7860 / J C D R / 2014 / 9517.5046$

21. Camilleri J, Motesin FE, Papaioannou S, et al. Biocompatibility of two commercial forms of mineral trioxide sggregate. Int Endod J 2004;37(10):699-704. DOI: 10.1111/j.1365-2591.2004. 00859.x.

22. Hashem AA, Hassanien EE. ProRoot MTA, MTA angelus and IRM used to repair large furcation perforations: sealability study. J Endod 2008;34(1):59-61. DOI: 10.1016/j.joen.2007.09.007.

23. Taylor MJ, Lynch E. Review microleakage. J Dent 1992;20(1):3-10. DOI: 10.1016/0300-5712(92)90002-T.

24. www.Spectrophotometer.com accessed on 5/01/2010, 8:15 pm.

25. Krupalini KS, Udayakumar, Jayalakshmi KB. A comparative evaluation of medicated calcium sulphate, hydroxylapatite, mineral trioxide aggregate as barrier and their effect on the sealing ability of furcation perforation repair material - an in vitro study. Int J Dent Res 2003;14(3):156-161. 